\title{
Optimization of neuronal morphologies for pattern recognition
}

\author{
Giseli de Sousa*, Reinoud Maex, Rod Adams, Neil Davey, Volker Steuber \\ From Nineteenth Annual Computational Neuroscience Meeting: CNS*2010 \\ San Antonio, TX, USA. 24-30 July 2010
}

Previous studies have shown that the morphology of a neuron can affect its firing pattern $[1,2]$. Specifically, some neuronal morphologies tend to favour bursting, where short sequences of spikes are interspersed with pauses in firing $[1,2]$. This type of bursting behaviour has been observed in cerebellar Purkinje cells (PCs), and previous work on associative memory in PCs has shown that the generation of burst-pause sequences can be important for information storage in the cerebellum [3]. These results have implications for the coding of information in the brain, but they are specific to one particular neuron with a highly specialised morphology. In this study we therefore use a general approach to optimise generic neuronal structures for pattern recognition, while analysing how their morphology influences their firing pattern.

To study how the ability of a neuron to perform pattern recognition depends on morphology, we have built a genomic representation of neuronal models, focusing as a first objective on optimising dendritic architectures. The optimization process uses an evolutionary algorithm and involves four steps. Firstly, genotypes are generated, which specify binary tree structures [4]. Secondly, the genotype is expressed as a model neuron phenotype, in which the branching pattern is derived from the genotype, and which is then converted to a multi-compartmental model written in NEURON simulation code. Thirdly, the fitness values are assessed by evaluating the pattern recognition performance. Finally, genetic variation is introduced, using a process where the genes are modified by crossover and mutation operators. Unlike previous work that focussed on generating a subset of realistic neuronal morphologies for specific computational tasks [5], our representation ensures that the

* Correspondence: g.sousa@herts.ac.uk

Science and Technology Research Institute, University of Hertfordshire,

Hatfield, Herts, AL10 9AB, UK algorithm can generate the set of all possible morphologies for a specific number of terminal branches. The fitness function evaluates pattern recognition performance as described previously [3,6], by storing a number of input patterns based on changing synaptic weights and quantifying the ability of the model to distinguish the set of stored patterns from a set of novel patterns. The discrimination between stored and novel patterns is evaluated for different features of the spike response and quantified by calculating a signal-to-noise ratio. The evolved artificial neuronal morphologies are compared with reconstructed morphologies from real neurons. An extension of the work involves optimizing other neuronal features such as types and distributions of ion channels and the spatial structure of inputs in patterns.

Published: 20 July 2010

\section{References}

1. Mainen ZF, Sejnowski TJ: Influence of dendritic structure on firing pattern in model neocortical neurons. Nature 1996, 382:363-366.

2. van Ooyen A, Duijnhouwer J, Remme M, van Pelt J: The effect of dendritic topology on firing patterns in model neurons. Network: Computation in Neural Systems 2002, 13:311-325.

3. Steuber V, Mittmann W, Hoebeek FE, Silver RA, De Zeeuw Cl, Häusser M, De Schutter E: Cerebellar LTD and Pattern Recognition by Purkinje Cells. Neuron 2007, 54(1):121-136.

4. Van Pelt J, Uylings HBM, Verwer RWH, Pentney RJ, Woldenberg MJ: Tree asymmetry-A sensitive and practical measure for binary topological trees. Bulletin of Mathematical Biology 1992, 54(5):759-784.

5. Stiefel KM, Sejnowski TJ: Mapping Function Onto Neuronal Morphology. J Neurophysiol 2007, 98(1):513-526.

6. de Sousa G, Adams R, Davey N, Maex R, Steuber V: The Effect of Different Forms of Synaptic Plasticity on Pattern Recognition in the Cerebellar Cortex. Adaptive and Natural Computing Algorithms 2009, 413-422.

\section{doi:10.1186/1471-2202-11-S1-P80}

Cite this article as: de Sousa et al:: Optimization of neuronal morphologies for pattern recognition. BMC Neuroscience 2010 11(Suppl 1):P80. 